\title{
QCD DIPOLE PREDICTION FOR DIS AND DIFFRACTIVE STRUCTURE FUNCTIONS
}

\author{
Ch.ROYON \\ DAPNIA-SPP, Centre d'Etudes de Saclay, F-91 191 Gif-sur-Yvette Cedex, France
}

\begin{abstract}
The $F_{2}, F_{G}, R=F_{L} / F_{T}$ proton structure functions are derived in the QCD dipole picture of BFKL dynamics. We get a three parameter fit describing the $1994 \mathrm{H} 1$ proton structure function $F_{2}$ data in the low $x$, moderate $Q^{2}$ range. Without any additional parameter, the gluon density and the longitudinal structure functions are predicted.The diffractive dissocjation processes are also discussed, and a new prediction for the proton diffractive structure function is obtained.
\end{abstract}

\footnotetext{
${ }^{a}$ Invited talk given at the 28th International Conference on High Energy Physics (ICHEP 96), 25-31 July 1996, Warsaw, Poland
}

The purpose of this contribution is to show that the QCD dipole picture 1 which contains the BFKL dynamics provides a pertinent model for describing the proton structure function at HERA in the low $x$ and moderate $Q^{2}$ range, as well as the diffractive structure function. The recently published $\mathrm{H} 1$ data might provide an opportunity to distinguish between the different QCD evolytion equations (DGLAP and BFKL equations 2) for small $x$ physics. This is why it is very important to test the BFKL dynamics using the dipole model and $k_{T}$ factorisation 3 . We can then get predictions for $F_{2}, F_{G}$, and $R=F_{L} / F_{T}$ proton structure functions, and for the proton diffractive structure functions which represents a new prediction of the dipole model.

\section{BFKL dynamics in the QCD dipole frame- work}

To obtain the structure function $F_{2}$, we use the $k_{T}$ factorisation theorem which is valid at high energy $(\operatorname{small} x)$. In a first step, we calculate the deep inelastic cross section $\sigma^{\text {onium }}$ of a photon of virtuality $Q^{2}$ on an onium state (heavy $q \bar{q}$ state). This onium sfate can be described by dipole configurations 1 . The photon-onium cross-section reads $\sigma^{\text {onium }}=\int d^{2} r d z \Phi^{(0)}(r, z) \sigma\left(x, Q^{2} ; r\right)$ where $\Phi^{(0)}$ is the probability distribution of dipole configurations of transverse coordinate $r$. In the $k_{T^{-}}$ factorization scheme $\mathrm{B}$, one writes

$$
\begin{aligned}
& Q^{2} \sigma\left(x, Q^{2} ; r\right)= \\
& \int^{Q^{2}} d^{2} \vec{k} \int_{0}^{1} \frac{d z}{z} \quad \hat{\sigma}\left(x / z, \vec{k}^{2} / Q^{2}\right) F\left(z,(k r)^{2}\right)
\end{aligned}
$$

where $\hat{\sigma} / Q^{2}$ is the $(\gamma g(k) \rightarrow q \bar{q})$ Born cross section for an off-shell gluon of transverse momentum $\vec{k} . F\left(z,(k r)^{2}\right)$ is the unintegrated gluon distribution of an onium state of size $r$ and contains the physics of the BFKL pomeron.

Using once more the $k_{T}$ factorisation in extracting a gluon from a dipole of transverse radius $r$ and assuming renormalisation group properties of $F$, one can show that

$$
\begin{gathered}
F^{\text {proton }}\left(x, Q^{2} ; Q_{0}^{2}\right)= \\
2 \frac{\bar{\alpha} N_{c}}{\pi} \int \frac{d \gamma}{2 i \pi} h(\gamma) \frac{v(\gamma)}{\gamma} w(\gamma)\left(\frac{Q^{2}}{Q_{0}^{2}}\right)^{\gamma} e^{\frac{\bar{\alpha} N_{c}}{\pi}} \chi(\gamma) \ln \left(\frac{1}{x}\right)
\end{gathered}
$$

where $\omega$ can be interpreted as the Mellin transformed probability of finding an onium of transverse mass $M^{2}$ in the proton, and $Q_{0}^{2}$ is a typically non perturbative proton scale.

We can use this generic result to get some predictions for $F_{T}, F_{L}$, and $F_{G}$ (respectively the transverse, longitudinal, and gluon structure functions) corresponding to $h_{T}, h_{L}$, and $h_{G}$ :

$$
\begin{aligned}
\left(\begin{array}{l}
h_{T} \\
h_{L} \\
h_{G}
\end{array}\right)=\frac{\bar{\alpha}}{3 \pi \gamma} \frac{(\Gamma(1-\gamma) \Gamma(1+\gamma))^{3}}{\Gamma(2-2 \gamma) \Gamma(2+2 \gamma)} \frac{1}{1-\frac{2}{3} \gamma} . \\
\left(\begin{array}{c}
(1+\gamma)\left(1-\frac{\gamma}{2}\right) \\
\gamma(1-\gamma) \\
1
\end{array}\right)
\end{aligned}
$$

The integral in $\gamma$ is performed by the steepest descent method. The saddle point is at $\gamma_{C}=$ $\frac{1}{2}\left(1-a \ln \frac{Q}{Q_{0}}\right)$ where $a=\left(\frac{\bar{\alpha} N_{c}}{\pi} 7 \zeta(3) \ln \frac{1}{x}\right)^{-1}$ It can be shown that the considered approximation is valid when $\ln Q / Q_{0} / \ln (1 / x)<<1$, that is small $x$, moderate $Q / Q_{0}$, kinematical domain. We finally obtain:

$$
F_{2} \equiv F_{T}+F_{L}
$$




$$
=C a^{1 / 2} e^{\left(\alpha_{P}-1\right) \ln \frac{1}{x}} \frac{Q}{Q_{0}} e^{-\frac{a}{2} \ln ^{2} \frac{Q}{Q_{0}}}
$$

where $\alpha_{P}-1=\frac{4 \bar{\alpha} N_{C} \ln 2}{\pi} . C, \alpha_{P}$ and $Q_{0}$ will be taken as free parameters for the fit of the H1 data, it will then be possible to compare with the values of $\alpha_{P}$ predicted by theory. We get finally $R$, and $F_{G} / F_{2}$, which are independent of the overall normalisation $C$ :

$$
\begin{gathered}
\frac{F_{G}}{F_{2}}=\left.\frac{1}{h_{T}+h_{L}}\right|_{\gamma=\gamma_{c}} \\
\equiv \frac{3 \pi \gamma_{c}}{\bar{\alpha}} \frac{1-\frac{2}{3} \gamma_{c}}{1+\frac{3}{2} \gamma_{c}-\frac{3}{2} \gamma_{c}^{2}} \frac{\Gamma\left(2-2 \gamma_{c}\right) \Gamma\left(2+2 \gamma_{c}\right)}{\left(\Gamma\left(1-\gamma_{c}\right) \Gamma\left(1+\gamma_{c}\right)\right)^{3}} \\
R=\frac{h_{L}}{h_{T}}\left(\gamma_{c}\right)=\frac{\gamma_{c}\left(1-\gamma_{c}\right)}{\left(1+\gamma_{c}\right)\left(1-\frac{\gamma_{c}}{2}\right)}
\end{gathered}
$$

where $\gamma_{C}$ is the saddle point value.

\section{$2 \quad F_{2}$ fit and prediction for $F_{G}$ and $R$}

In order to test the accuracy of the $F_{2}$ parametrisation obtained in formula (4), a fit using the recently published data from the $\mathrm{H} 1$ experiment $\mathrm{E}$ has been performed 0 . We have only used the points with $Q^{2} \leq 150 \mathrm{GeV}^{2}$ to remain in the domain of validity of the QCD dipole model. The $\chi^{2}$ is 101 for 130 points, and the values of the parameters are $\alpha_{P}=1.282, Q_{0}=0.63 \mathrm{GeV}, C=0.077$. The result of the fit is shown in figure 1a. The corresponding effective coupling constant to the obtained value of $\alpha_{P}$ is $\bar{\alpha}=0.11$, close to $\alpha\left(M_{Z}\right)$ used in the H1 QCD fit. The value of $Q_{0}$ corresponds to a tranverse size of $0.3 \mathrm{fm}$ which is the expected non perturbative scale.

Relation (5) provides a paramater-free prediction for the gluon density (not shown in the figure) which is in good agreement with the results obtained by the H1 QCD fits based on a NLO DGLAP evolution equation $\mathrm{E}$. We also give a prediction for the value of $R$, which is given in figure $1 b$. The only parameters which enters this prediction is $Q_{0}$, determined by the $F_{2}$ fit. The corresponding curve (full line) is compared with the one loop approximation of the $h$ functions (dashed curve) of formula (3). The comparison of the two curves exhibits the $(\ln 1 / x)$ terms resummation effects on the coefficient functions $h_{T}$ and $h_{L}$. The measurement of $R$ might be an opportunity to distinguish between the BFKL and DGLAP mechanisms, $\mathrm{R}$ being expected to be higher with the DGLAP mechanism. We thus think that a measurement of $R$ in this region would be useful.

\section{Hard diffraction in the QCD dipole model:}

The success of the dipole model applied to the proton structure function suggests to extend the investigations to other inclusive processes, in particular to diffractive dissociation. We can distinguish two different components:

- the "elastic" term which represents the elastic scattering of the onium on the target proton - the "triple-pomeron" term which represents the sum of all dipole-dipole interactions (it is dominant at large masses of the excited system).

Let us describe in more details each of the two components. The "triple-pomeron" term dominates at low $\beta$, where $\beta$ is the ratio between Bjorken$x$ and $x p$, the proton momentum fraction carried by the "pomeron" 6. This component, integrated over $t$, the momentum transfer, is factorisable in a part depending only on $x p$ (flux factor) and on a part depending only on $\beta$ and $Q^{2}$ ("pomeron" structure function) 6 .

$$
\begin{array}{r}
F_{2}^{D(3)}\left(Q^{2}, x p, \beta\right)=\Phi(x p) F_{P}\left(Q^{2}, \beta\right) \\
\Phi(x p)=8 x p^{1-2 \alpha_{P}}\left(\bar{\alpha} N_{c} 7 \zeta(3) \ln \frac{1}{x p}\right)^{-3}
\end{array}
$$

The effective exponent (the slope of $\ln F_{2}^{D}$ in $\ln x p$ ) is found to be dependent on $x p$ and much smaller than the BFKL exponent due to the $\ln (x p)$ in the flux factor. $F$ is equivalent to the proton structure function at small $\beta$.

The elastic component behaves quite differently 6 . First it dominates at $\beta \sim 1$. It is also factorisable like the inelastic component, but with a different flux factor, which means that the sum of the two components will not be factorisable. The $\beta$ dependence is quite flat at large $\beta$, and this is due to the fact that there is an interplay between the longitudinal and transverse components. The sum remains almost independent of $\beta$, whereas the $R=F_{L} / F_{T}$ ratio is strongly $\beta$ dependent. Once more, a $R$ measurement now in diffractive processes will be an interesting way to distinguish the different models, as the dipole model predicts different $\beta$ and $Q^{2}$ behaviours from the others 9 .

The sum of the two components shown in figure 2 describes quite well the H1 data. There is no further fit of the data as we chose to take the different parameters $\left(Q_{0}, \alpha_{P}\right.$, and the normalisation factor) from the $F_{2}$ fit. The most striking point is that we describe quite well the factorisa- 
tion breaking due to the resumpation of the two components at low and large $\beta$. The full line is the sum of the two components, the dashed line the inelastic one (which dominates at low $\beta$ ), and the dotted line the elastic one (which dominates at high $\beta$ ).

\section{Acknowledgments}

The results described in the present contribution come from a fruitful collaboration with A.Bialas, H.Navelet, R.Peschanski and S.Wallon.

\section{References}

1. A.H.Mueller and B.Patel, Nucl. Phys. B425 (1994) 471., A.H.Mueller, Nucl. Phys. B437 (1995) 107., N.N.Nikolaev and B.G.Zakharov, Zeit. für. Phys. C49 (1991) 607., A.H.Mueller, Nucl. Phys. $\mathbf{B 4 1 5}$ (1994) 373.

2. G. Altarelli and G. Parisi, Nucl. Phys. B126 (1977) 298; V.N. Gribov and L.N. Lipatov, Sov. Journ. Nucl. Phys. 15 (1972) 438 and 675., V.S.Fadin et al. Phys. Lett. $\mathbf{B 6 0}$ (1975) 50; I.I.Balitsky and L.N.Lipatov, Sov.J.Nucl.Phys. 28 (1978) 822.

3. S.Catani, M.Ciafaloni and Hautmann, Phys. Lett. B242 (1990) 97; Nucl. Phys. B366 (1991) 135; J.C.Collins and R.K.Ellis, Nucl. Phys. B360 (1991) 3; S.Catani and Hautmann, Phys. Lett. B315 (1993) 157; Nucl. Phys. B427 (1994) 475

4. H1 coll., S.Aid et al. preprint DESY 96-039, March 1996

5. H.Navelet, R.Peschanski, Ch.Royon, and S.Wallon, DESY preprint 96-108, to be published in Phys.Lett.B, hep-ph/9605389, and references therein, H.Navelet, R.Peschanski, Ch.Royon, Phys.Lett., B366, (1996) 329.

6. A.Bialas, R.Peschanski, Phys. Lett. B378 (1996) 302 and references therein, A.Bialas, 2nd Cracow epiphany conference (1996)

7. A.Bialas, R.Peschanski, preprint hep-ph/9605298, subm. to Phys. Lett., and references therein

8. A.Bialas, R.Peschanski, C.Royon, to appear in Phys. Lett.

9. M.F.McDermott et al., Proceedings of the HERA workshop (1996) and references therein
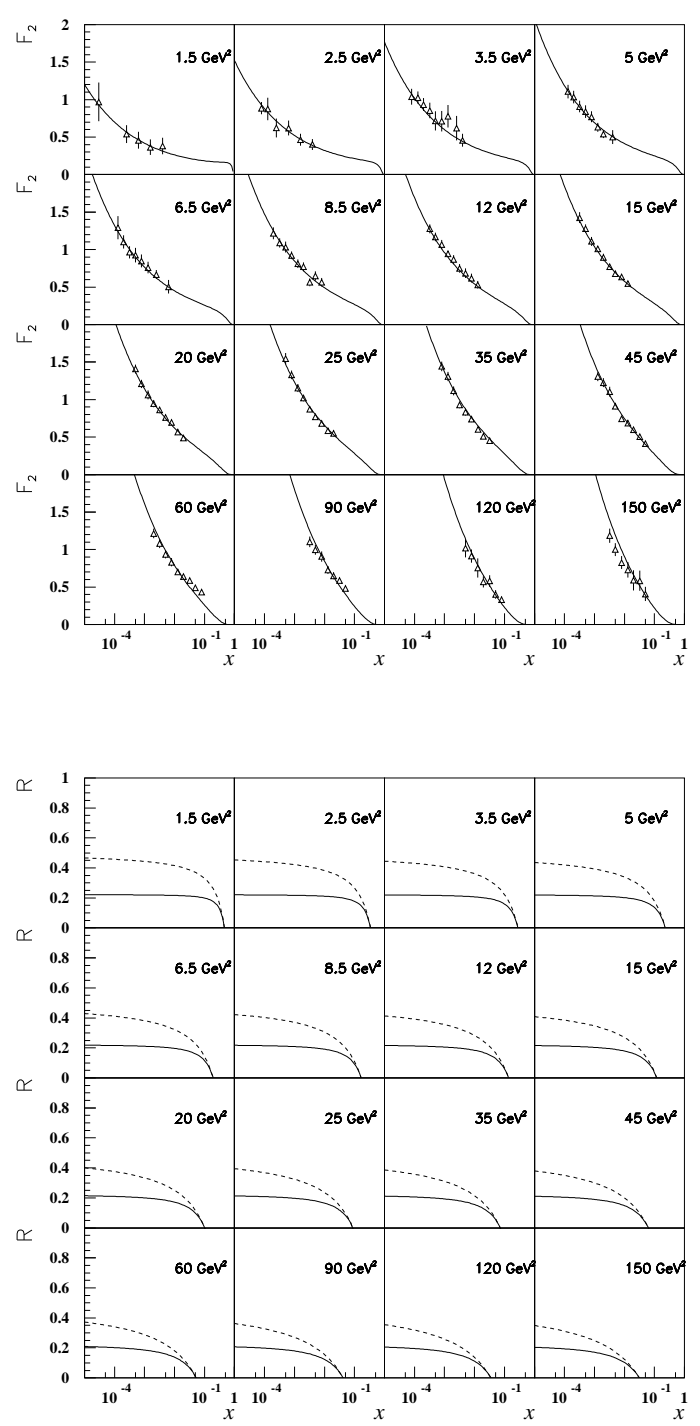

Figure 1: a: Results of the 3-parameter fit of the $\mathrm{H} 1$ proton structure function for $Q^{2} \leq 150 \mathrm{GeV}^{2}-\mathrm{b}$ :

Predictions on R (continuous line : resummed prediction) 


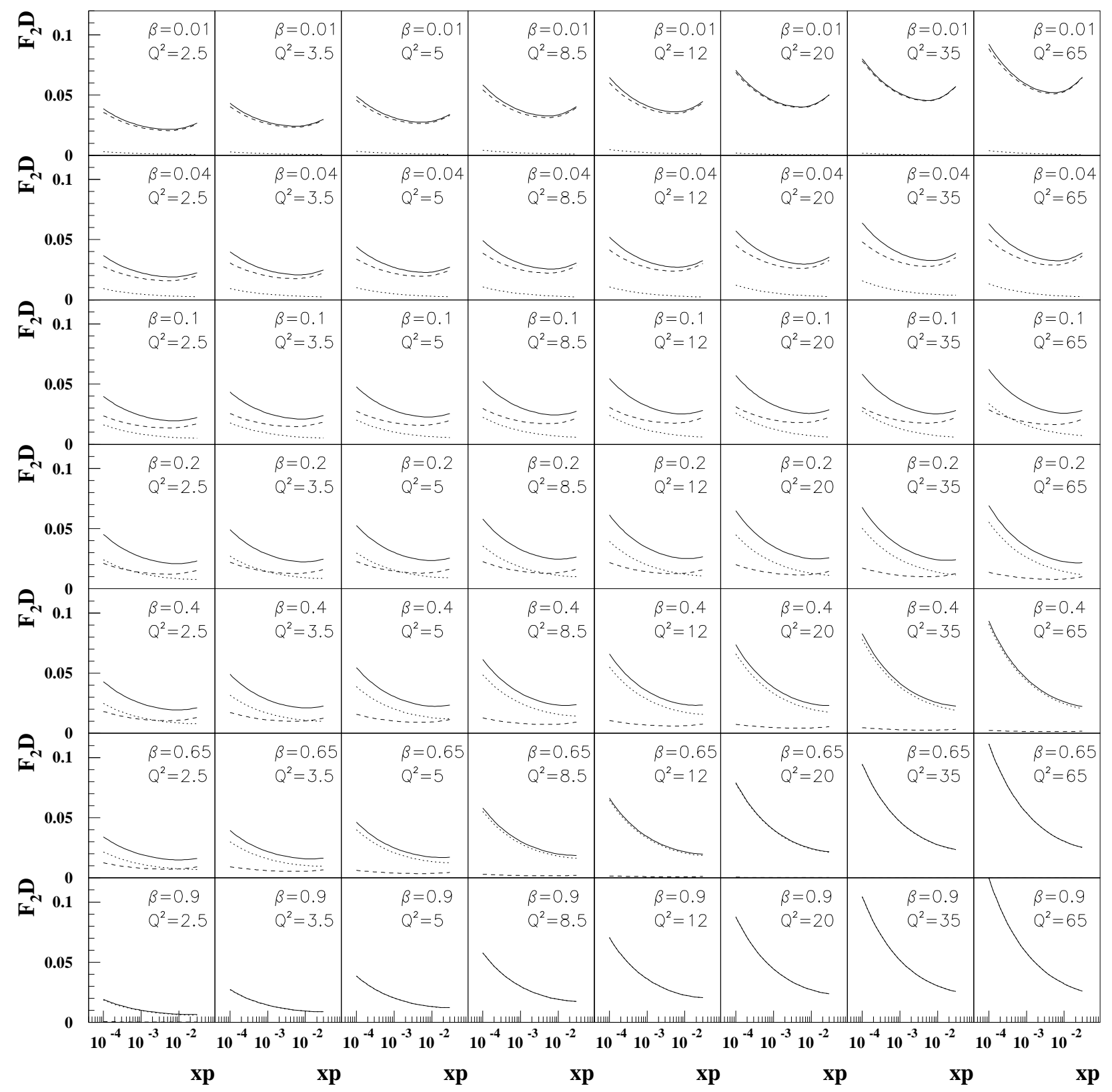

Figure 2: Prediction on F2D (cf text) 